\title{
Dissipativity Analysis of Descriptor Systems Using Image Space Characterization
}

\author{
Liang Qiao, ${ }^{1,2}$ Qingling Zhang, ${ }^{1,2}$ and Wanquan $\mathrm{Liu}^{3}$ \\ ${ }^{1}$ Institute of Systems Science, Northeastern University, Shenyang 110819, China \\ ${ }^{2}$ State Key Laboratory of Synthetical Automation for Process Industries, Northeastern University, China \\ ${ }^{3}$ Department of Computing, Curtin University, Perth, WA 6102, Australia
}

Correspondence should be addressed to Qingling Zhang; qlzhang@mail.neu.edu.cn

Received 20 March 2014; Revised 14 June 2014; Accepted 14 June 2014; Published 15 July 2014

Academic Editor: Masoud Hajarian

Copyright (C) 2014 Liang Qiao et al. This is an open access article distributed under the Creative Commons Attribution License, which permits unrestricted use, distribution, and reproduction in any medium, provided the original work is properly cited.

\begin{abstract}
In this paper, we analyze the dissipativity for descriptor systems with impulsive behavior based on image space analysis. First, a new image space is used to characterize state responses for descriptor systems. Based on such characterization and an integral property of delta function, a new necessary and sufficient condition for the dissipativity of descriptor systems is derived using the linear matrix inequality (LMI) approach. Also, some of the earlier related results on dissipativity for linear systems are investigated in the framework proposed in this paper. Finally, two examples are given to show the validity of the derived results.
\end{abstract}

\section{Introduction}

Since the notion of dissipative dynamical system was proposed by Willems in [1], it has played an important role in system analysis and synthesis of various control systems, such as in circuits, power systems, and mechanical systems $[1,2]$. In fact, this concept is an extension of passivity (or positive realness) and $H_{\infty}$ performance and is heavily used in stability analysis of nonlinear systems $[3,4]$. As we know, the concept of dissipativity in control systems generalizes several important results including the passivity theorem, bounded real lemma, Kalman-Yakubovich lemma, and the circle criterion $[4,5]$.

As to the importance of dissipativity, dissipative control for normal systems has attracted much attention [6-10]. With mild assumptions, the strict quadratic dissipativity of continuous/discrete normal systems is equivalent to their $H_{\infty}$ performance problem. Based on this observation, the existence conditions of feedback controllers to achieve asymptotic stability and strict dissipativity are derived, respectively, for continuous/discrete normal systems in $[6,7]$. In [8], the stability and strict dissipativity conditions for normal delay systems are investigated. In [9], the dissipativity conditions for symmetric normal systems are given. In [10], the optimal controller design is addressed for a class of normal systems which are dissipative with respect to a quadratic "power function."

There has been a growing research interest in descriptor systems in control community, since such systems can provide accurate representations for interconnected largescale systems, economic systems, electrical networks, power systems, and mechanical systems [11, 12]. Many important results for descriptor systems have been obtained in the last two decades, such as controllability and observability [13], $H_{2} / H_{\infty}$ control [14-16], positive realness [17, 18], and passive control [19] Dissipative control for descriptor systems has also attracted particular interest for its various applications in the literature [20-26]. In [20, 23], some necessary and sufficient conditions are given, respectively, to make continuous/discrete descriptor systems admissible and strictly dissipative. In [21], matrix inequalities are used to characterize necessary and sufficient conditions for dissipativity of descriptor systems. Based on results in [21], design of output feedback controllers to achieve dissipativity of the closedloop system is investigated in [22]. The dissipativity analysis for Takagi-Sugeno (T-S) fuzzy descriptor systems with timedelay is studied in [24]. In [25], the fuzzy controller and the fuzzy observer are given, which guarantees the closed loop T-S fuzzy descriptor system to be admissibility and 
strict $(Q, V, S)-\alpha$ dissipativity. In [26], the problem of delaydependent $\alpha$-dissipativity analysis is investigated for a general class of descriptor systems with Markovian jump parameters and mode-dependent mixed time-delays.

In fact, dissipative systems theory is linked to Lyapunov stability theory. There exist some tools from the dissipativity approach that can be used to construct some Lyapunov functions, which are related to $H_{\infty}$ control, positive realness, and so forth. So far, in [20-25], the dissipative analysis for descriptor systems is only for impulse-free systems. It is known that impulsive behavior is one main feature of descriptor systems being different from normal systems. In fact, these results are essentially the mild generalization of dissipativity theorems for normal systems, which do not reveal the nature of descriptor systems significantly. In [18], a new method of image space is proposed, and a necessary and sufficient condition for passivity and positive realness of descriptor system is given. As motivated by [18], we also give a new image space expression to characterize the state responses of descriptor systems with impulsive behavior. Though the idea is similar, the derived condition in this paper is much more explicit with expression of smaller matrix of lower dimensions. Also, we solve the dissipativity problem here instead of the positive realness problem addressed in [18].

This image space presents a new approach for the expression of descriptor systems. As impulsive behavior is involved in the system, we deal with the delta function as an approximation of a series of normal functions for mathematical simplicity. As such, the integrability of a supply function $r(u(t), y(t))$ is considered for descriptor systems with impulsive behavior. One can find that there are some constraints for the supply function in this case, which are different from the existing results. Then, a design approach which ensures the integrability of a supply function $r(u(t), y(t))$ is constructed. Consequently, a necessary and sufficient condition for dissipativity of descriptor systems is derived in terms of LMIs. This result is valid regardless of the existence of impulsive behavior, which is significantly different from the existing results. Thirdly, an algorithm on how to effectively solve these nonstrict LMIs is presented; meanwhile, the earlier related results are discussed with obtained results. Finally, one practical example and one numerical example are given to demonstrate the effectiveness of the results in this paper.

The layout of this paper is organized as below. We will present some preliminary results on characterization of descriptor systems based on image space in Section 2. The dissipativity analysis is presented in Section 3. The relationship with typical previous results is investigated in Section 4. Some illustrative examples are given in Section 5 and the final conclusions are made in Section 6.

For convenience of presentation, we first give the following notations used in this paper.

Notations. Let $\mathbf{R}, \mathbf{R}^{+}$, and $\mathbf{C}$ denote the sets of real, nonnegative real, and complex numbers, respectively. The notation $\mathbf{R}^{m \times n}$ denotes the set of $m \times n$ matrices with real elements. Let $M$ and $N$ be matrices. The notation $\operatorname{col}(M, N)$ denotes the matrix obtained by stacking $M$ over $N$. The image of $M$ is denoted by im $M$ and kernel of $M$ by ker $M$. The determinant of $M$ is denoted by $\operatorname{det}(M)$ and $\operatorname{rank}$ of $M$ by $\operatorname{rank}(M)$. Let $P$ be a square matrix; it is said to be symmetric if $P=P^{\mathrm{T}}$. We say that $P$ (necessarily symmetric) is positive semidefinite if $v^{\mathrm{T}} P v \geq 0$ for all vectors $v$. We write $P \geq 0$ by meaning that $P$ is positive semidefinite. Negative semidefiniteness is defined in a similar fashion. The notation $\operatorname{deg}(*)$ is the degree of a polynomial. For a symmetric matrix represented blockwise, offdiagonal blocks are abbreviated with $*$; that is,

$$
\left[\begin{array}{ll}
X_{11} & X_{12} \\
X_{12}^{\mathrm{T}} & X_{22}
\end{array}\right]=\left[\begin{array}{cc}
X_{11} & X_{12} \\
* & X_{22}
\end{array}\right]=\left[\begin{array}{cc}
X_{11} & * \\
X_{12}^{\mathrm{T}} & X_{22}
\end{array}\right]
$$

The notation $M \stackrel{\omega}{\leq} 0$ with $\omega$ being a vector space stands for $\varrho^{\mathrm{T}} M \varrho \leq 0$ for all $\varrho \in \omega$. Let $A C, C^{\infty}$, and $C_{p}^{h-1}$ denote, respectively, the sets of absolutely continuous, smooth, and $(h-1)$ times piecewise continuously differentiable functions.

\section{Preliminaries}

Consider the following descriptor system:

$$
\begin{gathered}
E \dot{x}(t)=A x(t)+B u(t), \\
y(t)=C x(t)+D u(t),
\end{gathered}
$$

where $x(t) \in \mathbf{R}^{n}$ is the state, $u(t) \in \mathbf{R}^{m}$ is the input, $y(t) \in \mathbf{R}^{r}$ is the output, the matrices $E, A, B, C, D$ are of appropriate sizes, and $\operatorname{rank} E=r \leq n$. The following concepts for descriptor systems are essential and will be used throughout this paper.

Definition 1 (see [11]). System (2a) and (2b) is said to be regular if $\operatorname{det}(s E-A)$ is not identically zero. It is said to be impulse-free if $\operatorname{deg}(\operatorname{det}(s E-A))=\operatorname{rank} E$.

As we know from [11], only when system (2a) and (2b) is regular, it has unique solution. Usually this regularity condition is required for all control problems of descriptor systems. In this case, there exist two invertible matrices $M \in$ $\mathbf{R}^{n \times n}$ and $T \in \mathbf{R}^{n \times n}$ such that

$$
\begin{gathered}
\bar{E}=\operatorname{MET}=\left[\begin{array}{cc}
I & 0 \\
0 & N
\end{array}\right], \quad \bar{A}=\mathrm{MAT}=\left[\begin{array}{cc}
A_{1} & 0 \\
0 & I
\end{array}\right], \\
\bar{B}=\mathrm{MB}=\left[\begin{array}{l}
B_{1} \\
B_{2}
\end{array}\right], \quad \bar{C}=\mathrm{CT}=\left[\begin{array}{ll}
C_{1} & C_{2}
\end{array}\right],
\end{gathered}
$$

where $A_{1} \in \mathbf{R}^{n_{1} \times n_{1}}, B_{i} \in \mathbf{R}^{n_{i} \times m}$, and $C_{i} \in \mathbf{R}^{r \times n_{i}}, i=1,2$ and $n_{1}+n_{2}=n . N$ is nilpotent; that is, $N^{h}=0$ for some integer $h \geq 1$. Then the restricted equivalent form of system (2a) and (2b) can be, respectively, expressed as

$$
\begin{aligned}
& \dot{x}_{1}(t)=A_{1} x_{1}(t)+B_{1} u(t), \\
& N \dot{x}_{2}(t)=x_{2}(t)+B_{2} u(t),
\end{aligned}
$$

which is called the Weierstrass canonical form. In fact, the invertible matrices $M$ and $T$ may be not unique and the transformed system is unique in this canonical form. 
Throughout the paper, we are interested in the solutions of system (2a) and (2b) at an initial value $x_{0}$. Then, any trajectory of $(2 a)$ and (2b) is given by (see [27])

$$
\begin{gathered}
x_{1}(t)=e^{A_{1} t} x_{1}(0)+\int_{0}^{t} e^{A_{1}(t-\tau)} B_{1} u(\tau) d \tau, \\
x_{2}(t)=-\sum_{i=0}^{h-1} N^{i} B_{2} u^{(i)}(t)-x_{20}(t),
\end{gathered}
$$

where

$$
x_{20}(t)=\sum_{i=0}^{h-1} \delta^{(i-1)}(t) N^{i} x_{20}+\sum_{i=0}^{h-1} \sum_{k=0}^{i-1} N^{i} B_{2} \delta^{(k)} u_{0}^{(i-k-1)} .
$$

One can see that if $N \neq 0$, this system will have impulsive behavior for nonzero initial values $x_{20}, u_{0}^{i-k-1}$, and discontinuity $u^{(i)}(t)$. Meanwhile, we know there are no impulsive terms in substate $x_{1}(t)$ when $u(t) \in C_{p}^{h-1}$. For simplicity, we assume consistent initial values in this paper; that is, $x_{20}=0$ and $u_{0}^{(i-k-1)}=0$. In this case, the main impulsive nature of the system is characterized by $N \neq 0$ in (5b). That indicates that if $u^{(i)}(t)$ has a discontinuity point at $t=\tau, x_{2}(t)$ will have impulsive behavior. In fact, when $N=0$, the system will have no impulsive behavior and the initial jump may appear as discussed in [28].

In order to investigate the solution of systems (2a) and (2b) from a new perspective, we follow the technique in [18] and define the following two sets:

$$
\mathcal{S}=\{(x(t), u(t)) \mid(x(t), u(t)) \text { is a solution of system }
$$

$$
\left.(2 \mathrm{a}),(2 \mathrm{~b}), u(t) \in A C, u(t) \in C_{p}^{h}\right\},
$$

$\mathcal{S}_{s}=\{(x(t), u(t)) \mid(x(t), u(t))$ is a solution of system

$$
\left.(2 \mathrm{a}),(2 \mathrm{~b}), u(t) \in C^{\infty}\right\} \text {. }
$$

One can see that $\mathcal{S}_{s} \subset \mathcal{S}$. Further we have the following results.

Lemma 2. For system (2a) and (2b), there exist matrices $V \in$ $\mathbf{R}^{n \times l}, U \in \mathbf{R}^{m \times l}$, and $W \in \mathbf{R}^{n \times l}$ with $l=n+m$ and $E W=$ $A V+B U$ such that the following statements hold.

(i) If $(x(t), u(t)) \in \mathcal{S}$ is a solution,

$$
\operatorname{col}(\dot{x}(t), x(t), u(t)) \in \operatorname{im}\{\operatorname{col}(W, V, U)\} .
$$

(ii) If $\xi \in \operatorname{im}\{\operatorname{col}(W, V, U)\}$, there exists a smooth solution $(x(t), u(t)) \in \mathcal{S}_{s}$ such that

$$
\operatorname{col}(\dot{x}(1), x(1), u(1))=\xi,
$$

where

$$
\begin{gathered}
V=\left[\begin{array}{ccc}
I & 0 & 0 \\
0 & -B_{2} & N
\end{array}\right], \\
W=\left[\begin{array}{ccc}
A_{1} & B_{1} & 0 \\
0 & 0 & I
\end{array}\right], \\
U=\left[\begin{array}{lll}
0 & I & 0
\end{array}\right] .
\end{gathered}
$$

Proof. This proof is similar to Lemma 3.2 in [18]. We omit them here.

Remark 3. The above lemma describes descriptor systems with impulsive behavior in terms of an image space. The image space $\operatorname{im}\{\operatorname{col}(W, V, U)\}$ can include all impulsive solutions of system ( $2 \mathrm{a})$ and $(2 \mathrm{~b})$ with consistent initial values. If we take

$$
\dot{x}_{2}(t)=-\sum_{i=0}^{h-1} N^{i} B_{2} u^{(i+1)}(t)
$$

into (24), we can obtain Lemma 3.2 in [18]. In terms of the expression formulae, Lemma 2 in this paper is more explicit than description of Lemma 3.2 in [18]. In detail, the matrices involved in Lemma 2 have lower dimensions and thus are more computationally efficient. One can see from the main results of this paper in next section that this new characterization is more powerful in solving the dissipativity problem for descriptor systems. Instead, [18] is only concerned with the passivity problem, which is a special case of this paper

The next two lemmas will be used in the proof of main results in next section.

Lemma 4 (see [29]). Given a nilpotent matrix $N \in \mathbf{R}^{n \times n}$ and a symmetric matrix $W \in \mathbf{R}^{n \times n}, W \geq 0$. If there exists a symmetric matrix $V \in \mathbf{R}^{n \times n}, V \geq 0$ such that

$$
N^{T} V+V N=-N^{T} W N
$$

and then

$$
N^{T} W N=0, \quad N^{T} V N=0 .
$$

Definition 5. A state space model $(E, A, B, C, D)$ is a realization of a transfer function $H(s)$ if $C(s E-A)^{-1} B+D=H(s)$. A realization $(E, A, B, C, D)$ of a transfer function $H(s)$ is said to be minimal if no other realization of $H(s)$ has smaller dimension.

In order to investigate the dissipativity of descriptor systems, we need one integral property of delta function. As we know, delta function is a generalized function and its properties can be investigated along the generalized function theory as in [30]. For mathematical simplicity and rigorousness, we treat this problem from engineering intuition by taking the delta function as an approximation of a series of normal functions as was done in [31]. We will investigate its generic correctness in the future.

Lemma 6 (see [31]). If the Dirac delta function $\delta(t)$ is defined as a limit of the following $g_{\varepsilon}(x)$ as $\varepsilon$ goes to zero, then it is not square integrable. 
Proof. As $\delta(t)$ is defined as the limit of the following $g_{\varepsilon}(x)$ as $\varepsilon$ goes to zero, then, for any continuous function $f(t)$, one has the following properties:

$$
\begin{gathered}
\delta(t)=0, \quad t \neq 0, \\
\int_{-\infty}^{\infty} \delta(t) d t=1, \\
\int_{-\infty}^{\infty} f(t) \delta(t) d t=f(0),
\end{gathered}
$$

where $f(0)$ is the function $f(t)$ at $t=0$ and

$$
g_{\varepsilon}(x)= \begin{cases}\frac{1}{\varepsilon^{2}}(x+\varepsilon), & -\varepsilon \leq x \leq 0 \\ \frac{1}{\varepsilon^{2}}(\varepsilon-x), & 0 \leq x \leq \varepsilon\end{cases}
$$

One can prove easily that as $\varepsilon$ goes to zero, $g_{\varepsilon}(x)$ will satisfy the above properties and it can approximate the Dirac delta function. This $g_{\varepsilon}(x)$ function is integrable, and the integral of this function is 1 which is independent of the value of $\varepsilon$. In fact, this integral can be computed explicitly (noting the integral symmetry) as

$$
\int_{-\infty}^{\infty} g_{\varepsilon}(x) d x=\frac{2}{\varepsilon^{2}} \int_{0}^{\varepsilon}(\varepsilon-x) d x=\frac{2}{\varepsilon^{2}}\left[x \varepsilon-\frac{x^{2}}{2}\right]_{0}^{\varepsilon}=1 .
$$

Now, we can compute the integral of the square of this function as follows (again, noting the symmetry of this function):

$$
\begin{aligned}
& \int_{-\infty}^{\infty} g_{\varepsilon}^{2}(x) d x \\
& \quad=\frac{2}{\varepsilon^{4}} \int_{0}^{\varepsilon}(\varepsilon-x)^{2} d x=\frac{2}{\varepsilon^{4}}\left[x \varepsilon^{2}-x^{2} \varepsilon+\frac{x^{3}}{3}\right]_{0}^{\varepsilon}=\frac{2}{3 \varepsilon}
\end{aligned}
$$

In the limit as $\varepsilon \rightarrow 0$, the integral of the square of this function approaches infinity. Thus, the Dirac delta function is not square integrable. This completes the proof.

There are different types of approximations for delta function by using different normal functions. Another typical one is using Gaussian function [32]. In fact, Lemma 6 is also true for the case of Gaussian approximation. With the approximation technique, we can obtain the following corollary.

Corollary 7. If $\alpha$ is a constant variable, and the function $\delta(t) \alpha \delta(t)$ with $\delta(t)$ defined as a limit of $g_{\varepsilon}(x)$ is Lebesgue integrable, then we have $\alpha=0$.

With the above property, we can prove the following lemma, which will play an important role in the proof of our main result in next section.
Lemma 8. If the function $\eta^{T} \Gamma \eta$ is Lebesgue integrable on $(-\infty,+\infty)$ for $\forall u \in C_{p}^{h-1}$, where

$$
\eta=\left[\begin{array}{c}
u(t) \\
\dot{u}(t) \\
\vdots \\
u^{(h-1)}(t)
\end{array}\right], \quad \Gamma=\left[\begin{array}{cccc}
\Gamma_{0} & 0 & \cdots & 0 \\
0 & \Gamma_{1} & \cdots & 0 \\
\vdots & \vdots & \ddots & \vdots \\
0 & 0 & \cdots & \Gamma_{h-1}
\end{array}\right] \text {, }
$$

then we have

$$
\Gamma_{i}=0, \quad i=1,2, \ldots, h-1 .
$$

Proof. Because $\eta^{\mathrm{T}} \Gamma \eta$ is Lebesgue integrable on $(-\infty,+\infty)$ for all $u \in C_{p}^{h-1}$, we can specially choose $u(t)$ to meet that $u^{(i)}(t)$, $i=0,1, \ldots, h-2$ are the Lebesgue integrable functions and $u^{(h-1)}(t)=\delta(t)$. By Corollary 7, we can obtain $\Gamma_{h-1}=0$. Then, we can specially choose $u(t)$ iteratively such that $u^{(i)}(t)$, $i=0,1, \ldots, h-3$ are the Lebesgue integrable functions and $u^{(h-2)}(t)=\delta(t)$. We will have $\Gamma_{h-2}=0$. Iteratively, we can prove that $\Gamma_{i}=0, i=1,2, \ldots, h-1$. This completes the proof.

\section{Dissipativity Analysis}

Following the intuition of dissipativity for a dynamic system given in [1], one can see that dissipativity of a dynamic implies that the internal energy storage of a system never exceeds the energy storage supplied to the system. In this paper, we formulate the notion of dissipativity for the system (2a) and (2b) via the so-called dissipativity inequality.

Definition 9. System (2a) and (2b) with supply rate $r(u(t)$, $y(t))$ which is locally Lebesgue integrable independently of the input and the initial conditions is said to be dissipative if there exists a nonnegative function $V(x(t)): \mathbf{R}^{n} \rightarrow \mathbf{R}^{+}$ (called the storage function) such that

$$
V\left(x\left(t_{1}\right)\right) \leq V\left(x\left(t_{0}\right)\right)+\int_{t_{0}}^{t_{1}} r(u(s), y(s)) d s
$$

for all $t_{0}, t_{1}$ with $t_{1} \geq t_{0}$ and the solution $(x(t), u(t))$ of system (2a) and (2b). If the above inequality is strict, then the system is said to be strictly dissipative.

Furthermore, the energy supply function $r(u(t), y(t))$ of systems (2a) and (2b) is usually defined as

$$
r(u(t), y(t))=y^{\mathrm{T}}(t) Q y(t)+2 y^{\mathrm{T}}(t) S u(t)+u^{\mathrm{T}}(t) R u(t),
$$

where $Q, S$, and $R$ are real matrices with $Q$ and $R$ being symmetric. In this case, the dissipative system (2a) and (2b) is said to be $(Q, S, R)$ dissipative. In practice, the following assumptions are usually put on for the study of dissipativity.

Assumption 10. Consider $Q \leq 0$.

Remark 11. From Definition 9, we know that the above strict $(Q, S, R)$ dissipativity includes $H_{\infty}$ performance and passivity 
as special cases. For example, if $Q=-I, S=0$, and $R=$ $\gamma^{2} I,(20)$ reduces to an $H_{\infty}$ performance index. If $Q=0, S=$ $I$, and $R=0,(20)$ corresponds to the passivity or positive realness for systems as discussed in [18].

One can see from the above remark that dissipativity analysis is more general in comparison to $H_{\infty}$ performance, positive realness, and passivity analysis. Though there are some results on other problems for descriptor systems with impulsive behavior, to the best of our knowledge, the analysis for dissipativity is still not available in the existing literature.

In order to give the main result in the following theorem, we use the following notations for simplicity:

$$
\begin{gathered}
\Pi=R+D^{\mathrm{T}} S+S^{\mathrm{T}} D+D^{\mathrm{T}} Q D, \\
\Omega=C^{\mathrm{T}} S+C^{\mathrm{T}} Q D .
\end{gathered}
$$

3.1. Design Energy Supply Function $r(u(t), y(t))$. In order to investigate the impulsive behavior for system (2a) and (2b), we need to discuss the integrability of supply function $r(u(t), y(t))$. There is a significant difference for descriptor systems with or without impulsive behavior. First, we give the following theorem based on Lemma 8 .

Theorem 12. The supply function $r(u(t), y(t))$ of system (2a) and (2b) is Lebesgue integrable for all $u \in C_{p}^{h-1}$ if and only if

$$
\begin{aligned}
& \mathrm{QC}_{2} N^{i} B_{2}=0, \quad i=1,2, \ldots, h-1 \\
& S C_{2} N^{i} B_{2}=0, \quad i=2,3, \ldots, h-1 .
\end{aligned}
$$

Proof. To prove this theorem, we rewrite

$$
\begin{aligned}
r(u(t), y(t)) & =y^{\mathrm{T}}(t) Q y(t)+2 y^{\mathrm{T}}(t) S u(t)+u^{\mathrm{T}}(t) R u(t) \\
& =x_{1}^{\mathrm{T}}(t) C_{1}^{\mathrm{T}} Q C_{1} x_{1}(t)+x_{2}^{\mathrm{T}}(t) C_{2}^{\mathrm{T}} Q C_{2} x_{2}(t)
\end{aligned}
$$

$$
\begin{aligned}
& +2 x_{1}^{\mathrm{T}}(t) C_{1}^{\mathrm{T}} Q C_{2} x_{2}(t)+u^{\mathrm{T}}(t) \Pi u(t) \\
& +2 x_{2}^{\mathrm{T}}(t) C_{2}^{\mathrm{T}}(Q D+S) u(t) \\
& +2 x_{1}^{\mathrm{T}}(t) C_{1}^{\mathrm{T}}(Q D+S) u(t) .
\end{aligned}
$$

Sufficiency. From (23)-(24) and $x_{2}=-\sum_{i=0}^{h-1} N^{i} B_{2} u^{(i)}(t)$, we have

$$
\begin{aligned}
& r(u(t), y(t)) \\
& =\left[\begin{array}{c}
x_{1}(t) \\
u(t) \\
\dot{u}(t)
\end{array}\right]^{\mathrm{T}}\left[\begin{array}{ccc}
C_{1}^{\mathrm{T}} Q C_{1} & \Xi_{1} & 0 \\
* & \Xi_{2} & S^{\mathrm{T}} C_{2} N B_{2} \\
* & * & 0
\end{array}\right]\left[\begin{array}{c}
x_{1}(t) \\
u(t) \\
\dot{u}(t)
\end{array}\right], \\
& \Xi_{1}=C_{1}^{\mathrm{T}} S+C_{1}^{\mathrm{T}} Q\left(D-C_{2} B_{2}\right), \\
& \Xi_{2}=R+\left(D-C_{2} B_{2}\right)^{\mathrm{T}} \mathrm{Q}\left(D-C_{2} B_{2}\right)+\left(D-C_{2} B_{2}\right)^{\mathrm{T}} S \\
& +S^{\mathrm{T}}\left(D-C_{2} B_{2}\right) .
\end{aligned}
$$

And $x_{1}(t)$ and $u(t)$ are locally integrable Lebesgue functions, so the supply function is Lebesgue integrable.

Necessity. Assume that the supply function $r(u(t), y(t))$ of system (2a) and (2b) is Lebesgue integrable for all $u \in C_{p}^{h-1}$, and then we have that

$$
\begin{aligned}
& x_{2}^{\mathrm{T}}(t) C_{2}^{\mathrm{T}} Q C_{2} x_{2}(t) \\
& =\left(-\sum_{i=0}^{h-1} N^{i} B_{2} u^{(i)}(t)\right)^{\mathrm{T}} C_{2}^{\mathrm{T}} Q C_{2}\left(-\sum_{i=0}^{h-1} N^{i} B_{2} u^{(i)}(t)\right) \\
& =\left[\begin{array}{c}
u(t) \\
\dot{u}(t) \\
\vdots \\
u^{(h-1)}(t)
\end{array}\right]^{\mathrm{T}}\left[\begin{array}{c}
u(t) \\
\dot{u}(t) \\
\vdots \\
u^{(h-1)}(t)
\end{array}\right]
\end{aligned}
$$

is Lebesgue integrable, where

$$
\Gamma=\left[\begin{array}{cccc}
B_{2}^{\mathrm{T}} C_{2}^{\mathrm{T}} Q C_{2} B_{2} & B_{2}^{\mathrm{T}} C_{2}^{\mathrm{T}} Q N C_{2} B_{2} & \cdots & B_{2}^{\mathrm{T}} C_{2}^{\mathrm{T}} Q C_{2} N^{h-1} B_{2} \\
B_{2}^{\mathrm{T}} N^{\mathrm{T}} C_{2}^{\mathrm{T}} Q C_{2} B_{2} & B_{2}^{\mathrm{T}} N^{\mathrm{T}} C_{2}^{\mathrm{T}} Q C_{2} N B_{2} & \cdots & B_{2}^{\mathrm{T}} N^{\mathrm{T}} C_{2}^{\mathrm{T}} Q C_{2} N^{h-1} B_{2} \\
\vdots & \vdots & \ddots & \vdots \\
B_{2}^{\mathrm{T}}\left(N^{h-1}\right)^{\mathrm{T}} C_{2}^{\mathrm{T}} Q C_{2} B_{2} & \cdots & \cdots & B_{2}^{\mathrm{T}}\left(N^{h-1}\right)^{\mathrm{T}} C_{2}^{\mathrm{T}} Q C_{2} N^{h-1} B_{2}
\end{array}\right] .
$$

By Lemma 8, we can have

$$
B_{2}^{\mathrm{T}} N^{i \mathrm{~T}} C_{2}^{\mathrm{T}} Q C_{2} N^{i} B_{2}=0, \quad i=1,2, \ldots, h-1 .
$$

Recall that $Q \leq 0$; we can obtain

$$
Q C_{2} N^{i} B_{2}=0, \quad i=1,2, \ldots, h-1 .
$$

With similar reasoning, we can derive that

$$
\begin{aligned}
u^{\mathrm{T}}(t) S^{\mathrm{T}} C_{2} x_{2}(t) & =-u^{\mathrm{T}}(t) S^{\mathrm{T}} C_{2}\left(\sum_{i=0}^{h-1} N^{i} B_{2} u^{(i)}(t)\right) \\
& =-\sum_{i=0}^{h-1} u^{\mathrm{T}}(t) S^{\mathrm{T}} C_{2} N^{i} B_{2} u^{(i)}(t)
\end{aligned}
$$


is Lebesgue integrable. Then, we can obtain

$$
S^{\mathrm{T}} C_{2} N^{i} B_{2}=0, \quad i=2,3, \ldots, h-1 .
$$

This completes the proof.

Remark 13. One can see from the above proof that if the descriptor system is impulse-free (i.e., $N=0$ ), the supply function is always Lebesgue integrable. Otherwise, the selection of parameters $Q$ and $S$ has some restrictions as stated in the above theorem. However, if the descriptor system (2a) and (2b) is output impulsive-free (i.e., $C_{2} N=0$ ), the selection for parameters $(Q \leq 0, S, R)$ would be very flexible. If the delta function is in general case, we can put these constraints as a requirement to eliminate impulse in the storage function.

Based on the observations from previous sections, we can state our main results in this paper.

\subsection{Dissipativity Analysis}

Theorem 14. Consider system (2a) and (2b); the following statements are equivalent.

(1) System (2a) and (2b) is $(Q, S, R)$ dissipative.

(2) The following LMIs,

$$
\begin{gathered}
P=P^{T} \geq 0 \\
{\left[\begin{array}{ccc}
0 & P & 0 \\
* & -C^{T} Q C & -C^{T} S-C^{T} Q D \\
* & * & -\Pi
\end{array}\right] \stackrel{\omega}{\leq} 0,}
\end{gathered}
$$

have a common solution $P$, where $\omega=\operatorname{im}\left[W^{T}, V^{T}, U^{T}\right]^{T}$.

Proof. (1) $\Rightarrow$ (2).

Suppose that system (2a) and (2b) is $(Q, S, R)$ dissipative with the storage function given below:

$$
V(x)=x^{\mathrm{T}}(t) P x(t)
$$

where $P \in \mathbf{R}^{n \times n}, P \geq 0$. Let $(x(t), u(t)) \in \mathcal{S}_{s}$. From the dissipativity inequality, we can obtain

$$
\int_{t_{0}}^{t_{1}} \frac{d V(t)}{d t} d s \leq \int_{t_{0}}^{t_{1}} r(u(s), y(s)) d s
$$

for all $t_{1} \geq t_{0}$. Since all involved functions are smooth, we can derive

$$
r(u(t), y(t)) \geq \frac{d V(t)}{d t} .
$$

Let

$r(u(t), y(t))=y^{\mathrm{T}}(t) Q y(t)+2 u(t)^{\mathrm{T}} \mathrm{Q} y(t)+u^{\mathrm{T}}(t) \mathrm{Q} u(t)$.
Then, we can have

$$
\begin{aligned}
\frac{d V(t)}{d t}-y^{\mathrm{T}}(t) Q y(t)-2 u^{\mathrm{T}}(t) Q y(t)-u^{\mathrm{T}}(t) Q u(t) \\
=\dot{x}^{\mathrm{T}}(t) P x(t)+x^{\mathrm{T}}(t) P \dot{x}(t)-x^{\mathrm{T}}(t) C^{\mathrm{T}} Q C x(t) \\
-2 x^{\mathrm{T}}(t)\left(C^{\mathrm{T}} S+C^{\mathrm{T}} \mathrm{Q} D\right) u(t)-u^{\mathrm{T}}(t) \Pi u(t) \\
=\left[\begin{array}{l}
\dot{x}(t) \\
x(t) \\
u(t)
\end{array}\right]^{\mathrm{T}}\left[\begin{array}{ccc}
0 & P & 0 \\
* & -C^{\mathrm{T}} Q C & \Omega \\
* & * & -\Pi
\end{array}\right]\left[\begin{array}{l}
\dot{x}(t) \\
x(t) \\
u(t)
\end{array}\right] \leq 0
\end{aligned}
$$

for $t \in \mathbf{R}^{+}$. Substituting $t=1$, we obtain

$$
\left[\begin{array}{l}
\dot{x}(1) \\
x(1) \\
u(1)
\end{array}\right]^{\mathrm{T}}\left[\begin{array}{ccc}
0 & P & 0 \\
* & -C^{\mathrm{T}} Q C & \Omega \\
* & * & -\Pi
\end{array}\right]\left[\begin{array}{l}
\dot{x}(1) \\
x(1) \\
u(1)
\end{array}\right] \leq 0 .
$$

It follows from the claim (ii) of Lemma 2 that $P$ is a solution to the LMIs in (33)-(34).

$(2) \Rightarrow(1)$.

Suppose that the LMIs (33)-(34) admit a solution $P$. From the claim (i) of Lemma 2; we can derive the following:

$$
\left[\begin{array}{c}
\dot{x}(t) \\
x(t) \\
u(t)
\end{array}\right]^{\mathrm{T}}\left[\begin{array}{ccc}
0 & P & 0 \\
* & -C^{\mathrm{T}} Q C & \Omega \\
* & * & -\Pi
\end{array}\right]\left[\begin{array}{l}
\dot{x}(t) \\
x(t) \\
u(t)
\end{array}\right] \leq 0
$$

whenever $(x(t), u(t)) \in \mathcal{S}$. Clearly, this yields

$$
r(u(t), y(t)) \geq \frac{d V(t)}{d t} .
$$

By integrating (42) from $t_{0}$ to $t_{1}$, we obtain the dissipativity inequality. The proof is completed.

Remark 15. It should be mentioned that Theorem 14 is valid for descriptor system with impulsive behavior. Furthermore, those LMIs conditions aim to seek symmetric solutions, which is different from the typical lower triangular matrix solutions for dissipativity of descriptor systems without impulsive behavior as reported in [21].

Remark 16. We can also consider the stability of system (2a) and (2b) by using our approach. If $u(t)=0$, we can obtain $\dot{x}_{2}(t)=0$. In this case, (8) will change to the following:

$$
\left[\begin{array}{c}
\dot{x}_{1}(t) \\
\dot{x}_{2}(t) \\
x_{1}(t) \\
x_{2}(t)
\end{array}\right]=\left[\begin{array}{c}
A_{1} \\
0 \\
I \\
0
\end{array}\right] x_{1}(t) .
$$

Then, we can choose the Lyapunov function

$$
V(x(t))=x^{\mathrm{T}}(t) P x(t),
$$

with

$$
P=\left[\begin{array}{ll}
P_{11} & P_{12} \\
P_{12}^{T} & P_{22}
\end{array}\right]>0
$$


By this way, we can obtain the stability condition of system (2a) and (2b) as follows:

$$
\left[\begin{array}{c}
A_{1} \\
0 \\
I \\
0
\end{array}\right]^{\mathrm{T}}\left[\begin{array}{cccc}
0 & 0 & P_{11} & P_{12} \\
0 & 0 & P_{12}^{\mathrm{T}} & P_{22} \\
P_{11} & P_{12} & 0 & 0 \\
P_{12}^{\mathrm{T}} & P_{22} & 0 & 0
\end{array}\right]\left[\begin{array}{c}
A_{1} \\
0 \\
I \\
0
\end{array}\right]=A_{1}^{\mathrm{T}} P_{11}+P_{11}^{\mathrm{T}} A_{1}<0
$$

This is a known stability result for descriptor systems as reported in [29].

Next, we discuss a parametrization form for all possible solutions of the LMIs (33)-(34).

Theorem 17. If the LMIs (33)-(34) are solvable, all possible solutions must be given by

$$
P=\left[\begin{array}{cc}
P_{11} & 0 \\
0 & P_{22}
\end{array}\right],
$$

where $P_{11} \in \mathbf{R}^{n_{1} \times n_{1}}$ and $P_{22} \in \mathbf{R}^{n_{2} \times n_{2}}$ are with the following requirements.

(1) $P_{11}$ is a solution to the following LMIs:

$$
\begin{gathered}
P_{11}=P_{11}^{T} \geq 0 \\
{\left[\begin{array}{cc}
A_{1}^{T} P_{11}+P_{11} A_{1}-C_{1}^{T} Q C_{1} & P_{1} B_{1}-\Xi_{1} \\
* & -\Xi_{2}
\end{array}\right] \leq 0 .}
\end{gathered}
$$

(2) $P_{22}$ is a solution of

$$
\begin{gathered}
P_{22}=P_{22}^{T} \geq 0, \\
P_{22}\left[\begin{array}{l}
N \\
B_{2}
\end{array}\right]=\left[\begin{array}{c}
0 \\
-N^{T} C_{2}^{T} S
\end{array}\right] .
\end{gathered}
$$

Proof. Suppose that the LMIs (33)-(34) admit a solution $P$. Let $P$ be a symmetric positive semidefinite matrix and partitioned as

$$
P=\left[\begin{array}{ll}
P_{11} & P_{12} \\
P_{12}^{\mathrm{T}} & P_{22}
\end{array}\right]=P^{\mathrm{T}} \geq 0
$$

It can be verified that the following equation holds:

$$
\begin{gathered}
{\left[\begin{array}{l}
W \\
V \\
U
\end{array}\right]^{\mathrm{T}}\left[\begin{array}{ccc}
0 & P & 0 \\
* & -C^{\mathrm{T}} Q C & -C^{\mathrm{T}} S-C^{\mathrm{T}} \mathrm{Q} D \\
* & * & -\Pi
\end{array}\right]\left[\begin{array}{l}
W \\
V \\
U
\end{array}\right]} \\
\quad=\left[\begin{array}{ccc}
M_{11} & M_{12} & M_{13} \\
* & M_{22} & M_{23} \\
* & * & M_{33}
\end{array}\right]
\end{gathered}
$$

where

$$
\begin{gathered}
M_{11}=A_{1}^{\mathrm{T}} P_{11}+P_{11}^{\mathrm{T}} A_{1}-C_{1}^{\mathrm{T}} Q C_{1}, \\
M_{12}=P_{11} B_{1}-A_{1}^{\mathrm{T}} P_{12} B_{2}+C_{1}^{\mathrm{T}} Q C_{2} B_{2}-C_{1}^{\mathrm{T}} S-C_{1}^{\mathrm{T}} Q D, \\
M_{13}=P_{12}-A_{1}^{\mathrm{T}} P_{12} N-C_{1}^{\mathrm{T}} Q C_{2} N B_{2}, \\
M_{22}=-B_{2}^{\mathrm{T}} P_{12}^{\mathrm{T}} B_{1}-B_{1}^{\mathrm{T}} P_{12} B_{2}+\left(S^{\mathrm{T}}+D^{\mathrm{T}} Q\right) C_{2} B_{2} \\
+B_{2}^{\mathrm{T}} C_{2}^{\mathrm{T}}(S+Q D)-B_{2}^{\mathrm{T}} C_{2}^{\mathrm{T}} Q C_{2} B_{2}+\Pi, \\
M_{23}=-B_{2}^{\mathrm{T}} P_{22}+B_{1}^{\mathrm{T}} P_{12} N+B_{2}^{\mathrm{T}} C_{2}^{\mathrm{T}} Q C_{2} N \\
-S^{\mathrm{T}} C_{2} N-D^{\mathrm{T}} Q C_{2} N, \\
M_{33}=N^{\mathrm{T}} P_{22}+P_{22} N-N^{\mathrm{T}} C_{2}^{\mathrm{T}} Q C_{2} N .
\end{gathered}
$$

Suppose that (51) is negative semidefinite; we will have $M_{33} \leq 0$. Since $N$ is nilpotent matrix, $P_{22} \geq 0$, and $-Q \geq 0$, we can obtain $M_{33}=0$; that is, $N^{\mathrm{T}} P_{22}+P_{22} N=N^{\mathrm{T}} C_{2}^{\mathrm{T}} Q C_{2} N$. By Lemma 4, we obtain

$$
N^{\mathrm{T}} P_{22} N=0, \quad-N^{\mathrm{T}} C_{2}^{\mathrm{T}} Q C_{2} N=0 .
$$

With simple manipulations, we can see that $P_{22} N=0$ and $Q_{2} N=0$. Since $M_{33}=0$, it follows from matrix theory that all the elements on the corresponding row and column blocks must be zero. In other words,

$$
M_{13}=0, \quad M_{23}=0 .
$$

Then, we can derive that

$$
P_{12}=0, \quad B_{2}^{\mathrm{T}} P_{22}=-S^{\mathrm{T}} C_{2} N .
$$

Finally, the right-hand side of (51) with combination of (33)-(34) will give the following conclusion:

$$
\left[\begin{array}{cc|cc}
A_{1}^{\mathrm{T}} P_{11}+P_{11}^{\mathrm{T}} A_{1}-C_{1}^{\mathrm{T}} Q C_{1} & M_{12} & 0 & 0 \\
* & M_{22} & 0 & 0 \\
\hline 0 & 0 & 0 & 0 \\
0 & 0 & 0 & 0
\end{array}\right] \leq 0 .
$$

We can see that $P_{11}$ is a solution to LMIs (48). This completes the proof.

Remark 18. By Theorem 17, we can obtain

$$
\begin{aligned}
V(x) & =x^{\mathrm{T}} P x=x_{1}^{\mathrm{T}} P_{11} x_{1}+x_{1}^{\mathrm{T}} P_{12} x_{2}+x_{2}^{\mathrm{T}} P_{12}^{\mathrm{T}} x_{1}+x_{2}^{\mathrm{T}} P_{22} x_{2} \\
& =x_{1}^{\mathrm{T}} P_{11} x_{1}+u^{\mathrm{T}} B_{2}^{\mathrm{T}} P_{22} B_{2} u
\end{aligned}
$$

So, storage function of system (2a) and (2b) can be divided into two parts: storage function of slow subsystem (4a) and storage function of fast subsystems (4b). This conclusion is in agreement with actual states; that is, the overall descriptor system is dissipative can be regarded as two subsystems are dissipative, respectively. 
Since $x_{1}(t)$ and $u(t)$ are absolutely continuous functions, $V(x)$ is absolutely continuous function and satisfies

$$
V\left(x\left(t_{1}\right)\right)-V\left(x\left(t_{0}\right)\right)=\int_{t_{0}}^{t_{1}} \frac{d V(t)}{d t} d t .
$$

This reveals that (42) is Lebesgue integrable. By the above discussion and Theorem 12, if suitable matrices are chosen according to these theorems, the impulsive behavior does not display out the influence in the running process of the system, and the descriptor system is dissipative. That implies that the impulses have to be eliminated from (some parts of) the signals related to the storage function.

One will see that the main result in this paper is quite different from the existing results.

\section{Comparison with Existing Results}

In this section, we will compare the obtained results in the last section with some important existing results.

First, we compare with the results for normal systems. If $E=I$, one can verify the following equation easily:

$$
\omega=\operatorname{im}\left[\begin{array}{l}
W \\
V \\
U
\end{array}\right]=\operatorname{im}\left[\begin{array}{cc}
A & B \\
I & 0 \\
0 & I
\end{array}\right] .
$$

This will lead to the following result.

Corollary 19. The continuous normal system is $(Q, S, R)$ dissipative if and only if there exists $P \in \mathbf{R}^{n \times n}$ with $P=P^{T} \geq 0$ such that the following LMI is satisfied:

$$
\left[\begin{array}{cc}
A^{T} P+P A-C^{T} Q C & P B-\Omega \\
* & -\Pi
\end{array}\right] \leq 0 .
$$

Proof. When $E=I$, by using Theorem 14 and (59), we have

$$
\begin{gathered}
{\left[\begin{array}{cc}
A & B \\
I & 0 \\
0 & I
\end{array}\right]^{\mathrm{T}}\left[\begin{array}{ccc}
0 & P & 0 \\
* & -C^{\mathrm{T}} Q C & -\Omega \\
* & * & -\Pi
\end{array}\right]\left[\begin{array}{cc}
A & B \\
I & 0 \\
0 & I
\end{array}\right]} \\
\quad=\left[\begin{array}{cc}
A^{\mathrm{T}} P+P A-C^{\mathrm{T}} Q C & P B-\Omega \\
* & -\Pi
\end{array}\right] \leq 0 .
\end{gathered}
$$

Corollary 19 is proved.

Remark 20. If $E=I$, our theorems become necessary and sufficient conditions for dissipativity of continuous normal systems. In fact, Corollary 19 is equivalent to the dissipative lemma reported in [10].

Next, we compare with some results for descriptor systems. First, we derive the following results, which are actually new for descriptor systems.

Corollary 21. System (2a) and (2b) is $(Q, S, R)$ dissipative if there exists $X \in \mathbf{R}^{n \times n}$ such that the following LMIs are satisfied:

$$
\begin{gathered}
E^{T} X=X^{T} E \geq 0, \\
{\left[\begin{array}{cc}
A^{T} X+X^{T} A-C^{T} Q C & X^{T} B-\Omega \\
* & -\Pi
\end{array}\right] \leq 0 .}
\end{gathered}
$$

Proof. When system (2a) and (2b) is $(Q, S, R)$ dissipative, by taking $P=E^{\mathrm{T}} X$ and using $\mathrm{EW}=\mathrm{AV}+\mathrm{BU}$, we can obtain the following:

$$
\begin{aligned}
& {\left[\begin{array}{l}
W \\
V \\
U
\end{array}\right]^{\mathrm{T}}\left[\begin{array}{ccc}
0 & P & 0 \\
* & -C^{\mathrm{T}} Q C & -\Omega \\
* & * & -\Pi
\end{array}\right]\left[\begin{array}{l}
W \\
V \\
U
\end{array}\right]} \\
& =\left[\begin{array}{c}
V \\
U
\end{array}\right]^{\mathrm{T}}\left[\begin{array}{c|c}
A^{\mathrm{T}} X+X^{\mathrm{T}} A & X^{\mathrm{T}} B-\Omega \\
-C^{\mathrm{T}} Q C & \\
\hline * & -\Pi
\end{array}\right]\left[\begin{array}{c}
V \\
U
\end{array}\right] \leq 0 .
\end{aligned}
$$

From Theorem 14, one can see that Corollary 21 is proved.

Similarly, by taking $P=E^{\mathrm{T}} X E$ and $\mathrm{EW}=\mathrm{AV}+\mathrm{BU}$, we can obtain the following results.

Corollary 22. System (2a) and (2b) is $(Q, S, R)$ dissipative if there exists symmetric matrix $X \in \mathbf{R}^{n \times n}$ such that the following LMIs are satisfied:

$$
\begin{gathered}
E^{T} X E \geq 0 \\
{\left[\begin{array}{cc}
A^{T} X E+E^{T} X A-C^{T} Q C & E^{T} X^{T} B-\Omega \\
* & -\Pi
\end{array}\right] \leq 0 .}
\end{gathered}
$$

Though the LMIs (33) and (34) are investigated in general in the last section, we will give a special case in the next corollary, which can be used easily in practical computation.

Corollary 23. Suppose that

(1) $(E, A, B, C)$ is $(Q, S, R)$ dissipative;

(2) $(E, A, B, C)$ is minimal;

(3) $(E, A, B, C)$ is given in the Weierstrass form, and $N=$ $\left[\begin{array}{ll}0 & I \\ 0 & 0\end{array}\right]$.

Then, the LMIs (33)-(34) are solvable and all the solutions can be given by

$$
\widehat{P}=\left[\begin{array}{ccc}
\widehat{P}_{11} & 0 & 0 \\
0 & 0 & 0 \\
0 & 0 & \widehat{P}_{33}
\end{array}\right]
$$

with the following requirements:

(1) $\widehat{P}_{11}$ is a solution to the following LMIs:

$$
\begin{gathered}
\widehat{P}_{11}=\widehat{P}_{11}^{T} \geq 0 \\
{\left[\begin{array}{cc}
\widehat{A}_{1}^{T} \widehat{P}_{11}+\widehat{P}_{11} \widehat{A}_{1}-\widehat{C}_{1}^{T} Q \widehat{C}_{1} & \widehat{P}_{1} \widehat{B}_{1}-\widehat{\Xi}_{1} \\
* & -\widehat{\Xi}_{2}
\end{array}\right] \leq 0} \\
\text { where } \\
\widehat{\Xi}_{1}=\widehat{C}_{1}^{T}\left(S+Q\left(\widehat{D}-\widehat{C}_{2} \widehat{B}_{2}-\widehat{C}_{3} \widehat{B}_{3}\right)\right) \\
\widehat{\Xi}_{2}=R+\left(\widehat{D}-\widehat{C}_{2} \widehat{B}_{2}-\widehat{C}_{3} \widehat{B}_{3}\right)^{T} \mathrm{Q}\left(\widehat{D}-\widehat{C}_{2} \widehat{B}_{2}-\widehat{C}_{3} \widehat{B}_{3}\right) \\
+\left(\widehat{D}-\widehat{C}_{2} \widehat{B}_{2}-\widehat{C}_{3} \widehat{B}_{3}\right)^{T} S+S^{T}\left(\widehat{D}-\widehat{C}_{2} \widehat{B}_{2}-\widehat{C}_{3} \widehat{B}_{3}\right)
\end{gathered}
$$


(2) $\widehat{P}_{33}$ is the unique solution of $\widehat{B}_{3}^{T} \widehat{P}_{33}=-S^{T} \widehat{C}_{2}$.

Proof. By (2)-(3), we have

$$
\begin{aligned}
& (E, A, B, C) \\
& \quad=\left(\left[\begin{array}{lll}
I & 0 & 0 \\
0 & 0 & I \\
0 & 0 & 0
\end{array}\right],\left[\begin{array}{ccc}
\widehat{A}_{1} & 0 & 0 \\
0 & I & 0 \\
0 & 0 & I
\end{array}\right],\left[\begin{array}{l}
\widehat{B}_{1} \\
\widehat{B}_{2} \\
\widehat{B}_{3}
\end{array}\right],\left[\begin{array}{lll}
\widehat{C}_{1} & \widehat{C}_{1} & \widehat{C}_{3}
\end{array}\right]\right) .
\end{aligned}
$$

Let $\widehat{P}$ be a symmetric positive semidefinite matrix and partitioned as

$$
\widehat{P}=\left[\begin{array}{lll}
\widehat{P}_{11} & \widehat{P}_{12} & \widehat{P}_{13} \\
\widehat{P}_{12}^{\mathrm{T}} & \widehat{P}_{22} & \widehat{P}_{23} \\
\widehat{P}_{13}^{\mathrm{T}} & \widehat{P}_{23}^{\mathrm{T}} & \widehat{P}_{33}
\end{array}\right]
$$

where $\widehat{P}_{11} \in \mathbf{R}^{n_{1} \times n_{1}}, \widehat{P}_{22} \in \mathbf{R}^{l_{2} \times l_{2}}, \widehat{P}_{33} \in \mathbf{R}^{l_{3} \times l_{3}}$, and $l_{2}+l_{3}=n_{2}$. By Theorem 14, we have

$$
\begin{aligned}
& P_{11}=\widehat{P}_{11} \text {, } \\
& P_{12}=\left[\begin{array}{ll}
\widehat{P}_{12} & \widehat{P}_{13}
\end{array}\right]=0 \text {, } \\
& P_{22} N=\left[\begin{array}{ll}
\widehat{P}_{22} & \widehat{P}_{23} \\
\widehat{P}_{23}^{\mathrm{T}} & \widehat{P}_{33}
\end{array}\right]\left[\begin{array}{ll}
0 & I \\
0 & 0
\end{array}\right]=0 \text {, } \\
& B_{2}^{\mathrm{T}} P_{22}=\left[\begin{array}{l}
\widehat{B}_{2} \\
\widehat{B}_{3}
\end{array}\right]^{\mathrm{T}}\left[\begin{array}{ll}
\widehat{P}_{22} & \widehat{P}_{23} \\
\widehat{P}_{23}^{\mathrm{T}} & \widehat{P}_{33}
\end{array}\right] \\
& =S^{\mathrm{T}} C_{2} N=S^{\mathrm{T}}\left[\begin{array}{ll}
\widehat{C}_{2} & \widehat{C}_{3}
\end{array}\right]\left[\begin{array}{ll}
0 & I \\
0 & 0
\end{array}\right]=0 .
\end{aligned}
$$

Then, straightforward calculations yield

$$
\widehat{P}_{13}=0, \quad \widehat{P}_{13}=0, \quad \widehat{P}_{23}=0, \quad \widehat{P}_{22}=0, \quad \widehat{B}_{3}^{\mathrm{T}} \widehat{P}_{33}=-S^{\mathrm{T}} \widehat{C}_{2} .
$$

Due to minimality, $\widehat{B}_{3}$ must be full row rank. Hence, $\widehat{P}_{33}$ is the unique solution of $\widehat{B}_{3}^{\mathrm{T}} \widehat{P}_{33}=-S^{\mathrm{T}} \widehat{C}_{2}$.

Remark 24. By particularly choosing $Q=0, S=I$, and $R=0$, we can obtain corresponding results for Corollaries 21-23 and they are in fact the Theorems 6.4(1), 6.4(2), and 5.4 in [18].

Before we conclude this section, we discuss how to effectively solve the nonstrict LMIs (33)-(34). We can see that they are given with an image space and not the conventional strict LMIs. By (34), we know that

$$
\varrho^{\mathrm{T}}\left[\begin{array}{ccc}
0 & P & 0 \\
* & -C^{\mathrm{T}} Q C & -C^{\mathrm{T}} S-C^{\mathrm{T}} Q D \\
* & * & -\Pi
\end{array}\right] \varrho \leq 0
$$

for all $\varrho \in \omega=\operatorname{im}\left[\begin{array}{l}W \\ V \\ U\end{array}\right]$. This implies that LMI (34) is equivalent to

$$
\left[\begin{array}{c}
W \\
V \\
U
\end{array}\right]^{\mathrm{T}}\left[\begin{array}{ccc}
0 & P & 0 \\
* & -C^{\mathrm{T}} Q C & -C^{\mathrm{T}} S-C^{\mathrm{T}} Q D \\
* & * & -\Pi
\end{array}\right]\left[\begin{array}{c}
W \\
V \\
U
\end{array}\right] \leq 0
$$

According to the discussion above, LMIs (33) and (73) are also not the standard LMIs which can be solved by the LMI toolbox in MATLAB directly. Luckily, LMIs in (33) and (73) problem are still a semidefinite programming (SDP) problem. So, we can use MATLAB toolbox YALMIP [33] which was initially developed to model SDP and solved by interfacing external solver-SeDuMi [34]. YALMIP toolbox solves optimization problems in general and can be used for control oriented SDP problems. Next, we will give an algorithm for solving the nonstrict LMIs (33) and (73) in detail.

Step 1. Find two invertible matrices $M$ and $T$, and change system (2a) and (2b) into Weierstrass canonical forms.

Step 2. Compute the matrices $W, V, U$, and $Y=\left[\begin{array}{c}W \\ V \\ U\end{array}\right]$.

Step 3. Solve LMIs (33) and (73) as follows. Define decision variable and constraints

$$
P=\operatorname{sdpvar}(n, n), \quad F=\operatorname{set}(P \geq 0) .
$$

And let

$$
F=F+Y^{\mathrm{T}}\left[\begin{array}{ccc}
0 & P & 0 \\
* & -C^{\mathrm{T}} Q C & -C^{\mathrm{T}} S-C^{\mathrm{T}} \mathrm{QD} \\
* & * & -\Pi
\end{array}\right] Y \geq 0 .
$$

Then, solve problem solvesdp $(F)$ and $P=\operatorname{double}(P)$.

\section{Illustrative Examples}

In this section, we will present two examples to demonstrate the effectiveness and the merits of the proposed results.

Example 1 (see [30]). Consider a mechanical system shown in Figure 1. This system consists of two one-mass oscillators connected by a dashpot element, which is described by

$$
\begin{aligned}
M \ddot{z}+D \dot{z}+K z & =L f+J \mu, \\
G \dot{z}+H z & =0,
\end{aligned}
$$

where $z \in R^{n}$ is the displacement vector, $f \in R^{n}$ is the vector of known input forces, $\mu \in R^{q}$ is the vector of Lagrangian multipliers, $M$ is the inertial matrix, which is usually symmetric and positive definite, $D$ is the damping and gyroscopic matrix, $K$ is the stiffness and circulator matrix, $L$ is the force distribution matrix, $J$ is the Jacobian of the constraint equation, and $G$ and $H$ are the coefficient matrices of the constraint equation. All matrices in (76) are known and constant with appropriate dimensions.

Assume that a linear combination of displacements and velocities is measurable; that is, the output equation is of the form

$$
y=C_{p} z+C_{v} \dot{z}
$$




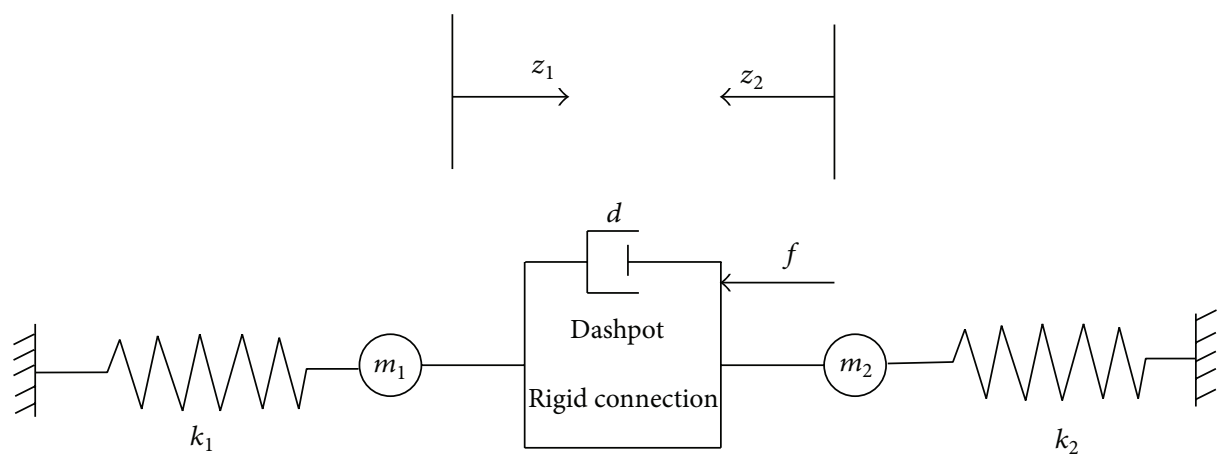

FIGURE 1: Two connected one-mass oscillators. as

By further choosing the state vector and the input vector

$$
x=\left(\begin{array}{c}
z \\
\dot{z} \\
\mu
\end{array}\right), \quad u=f
$$

respectively, we can rewrite the above (76)-(77) in the following descriptor system form. Let $m_{1}=1, m_{2}=1, d=1$, $k_{1}=2$, and $k_{2}=1$. Thus, we obtain a descriptor system with the following parameters:

$$
\begin{gathered}
E \dot{x}(t)=A x(t)+B u(t), \\
y(t)=C x(t),
\end{gathered}
$$

where

$$
\begin{aligned}
& E=\left[\begin{array}{lllll}
1 & 0 & 0 & 0 & 0 \\
0 & 1 & 0 & 0 & 0 \\
0 & 0 & 1 & 0 & 0 \\
0 & 0 & 0 & 1 & 0 \\
0 & 0 & 0 & 0 & 0
\end{array}\right], \quad A=\left[\begin{array}{ccccc}
0 & 0 & 1 & 0 & 0 \\
0 & 0 & 0 & 1 & 0 \\
-2 & 0 & -1 & -1 & 1 \\
0 & -1 & -1 & -1 & 1 \\
1 & 1 & 0 & 0 & 0
\end{array}\right], \\
& B=\left[\begin{array}{c}
0 \\
0 \\
-1 \\
1 \\
0
\end{array}\right], \quad C=\left[\begin{array}{lllll}
1 & 1 & 0 & 0 & 0
\end{array}\right]
\end{aligned}
$$

One can verify that $\operatorname{deg}(\operatorname{det}(s E-A))=2 \neq 4=\operatorname{rank} E$, so this descriptor system is not impulse-free. If we choose $Q=-1, S=1$, and $R=5$ in the energy supply function, one solution to the LMIs of Theorem 14 is as follows:

$$
P=\left[\begin{array}{ccccc}
0 & 0 & 0 & 0 & 0 \\
0 & 0 & 0 & 0 & 0 \\
0 & 0 & 0 & 0 & 0 \\
0 & 0 & 0 & 0.0001 & 0.0149 \\
0 & 0 & 0 & 0.0149 & 8.2588
\end{array}\right]
$$

Therefore, this system is dissipative although it is not impulsefree.
Remark 25. In the above example, we know that this descriptor system consists of the physical equations

$$
\begin{gathered}
\ddot{z}_{1}+\dot{z}_{1}+\dot{z}_{2}+2 z_{1}=-f+\mu \\
\ddot{z}_{2}+\dot{z}_{1}+\dot{z}_{2}+z_{2}=f+\mu
\end{gathered}
$$

with a constraint equation $z_{1}+z_{2}=0$. After substituting the constraint equation into the physical equations while ignoring its initial conditions, we can obtain the following system:

$$
\begin{gathered}
\ddot{z}_{1}+2 z_{1}=-f+\mu, \\
\ddot{z}_{2}+z_{2}=f+\mu .
\end{gathered}
$$

This becomes a normal system. According to [2], we know that this normal system is dissipative with energy function $V(z, \dot{z})=(1 / 2) m \dot{z}^{2}+(1 / 2) K z^{2}$ since

$$
V\left[z\left(t_{1}\right), \dot{z}\left(t_{1}\right)\right] \leq V\left[z\left(t_{0}\right), \dot{z}\left(t_{0}\right)\right]+\int_{t_{0}}^{t_{1}} F(t) v(t) d t
$$

where the force $F(t)=L f+J \mu$ and the velocity $v(t)=\dot{z}(t)$. Therefore, the original descriptor system is dissipative. This is consistent with Theorem 14.

The above example shows that a dynamic system can be modeled with different models/equations with different simplification requirements. The results obtained in this paper can be used to investigate the dissipativity problem for descriptor system in (76)-(77), while the existing results cannot be used due to the impulsive behavior. When we use the model (76)-(77), in fact we ignored the impulsive behavior due to the inconsistent initial conditions.

Next, we will demonstrate how to appropriately choose $(Q, S, R)$ to ensure that the supply function $r(y(t), u(t))$ is integrable.

Example 2. Consider a descriptor system

$$
\begin{gathered}
N \dot{x}(t)=A x(t)+B u(t), \\
y(t)=C x(t),
\end{gathered}
$$


where

$$
\begin{array}{cc}
N=\left[\begin{array}{lll}
0 & 1 & 0 \\
0 & 0 & 1 \\
0 & 0 & 0
\end{array}\right], & A=\left[\begin{array}{lll}
1 & 0 & 0 \\
0 & 1 & 0 \\
0 & 0 & 1
\end{array}\right], \\
B & =\left[\begin{array}{l}
-1 \\
-1 \\
-1
\end{array}\right],
\end{array}
$$

According to (3), its solution is given by

$$
x(t)=-\sum_{i=0}^{2} N_{i} B u^{(i)}(t)
$$

which gives

$$
\begin{aligned}
& x_{1}(t)=u(t)+\dot{u}(t)+\ddot{u}(t), \\
& x_{2}(t)=u(t)+\dot{u}(t), \\
& x_{3}(t)=u(t) .
\end{aligned}
$$

In this system, impulsive behavior may also appear in the state response, although the input function is continuous. And such behavior appears due to the discontinuous property in input derivatives. If we choose

$$
Q=\left[\begin{array}{cc}
0 & 0 \\
0 & -1
\end{array}\right] \leq 0, \quad S=\left[\begin{array}{l}
0 \\
1
\end{array}\right], \quad R=1
$$

which satisfy the condition of Theorem 12, then we can obtain

$$
\begin{aligned}
r(u(t), y(t))= & y^{\mathrm{T}}(t) Q y(t)+2 u(t)^{\mathrm{T}} S y(t)+u^{\mathrm{T}}(t) R u(t) \\
= & -x^{\mathrm{T}}(t) C^{\mathrm{T}} Q C x(t)+2 x^{\mathrm{T}}(t) C^{\mathrm{T}} S u(t) \\
& +u^{\mathrm{T}}(t) R u(t) \\
= & -x_{3}^{\mathrm{T}}(t) x_{3}(t)+x_{3}^{\mathrm{T}}(t) u(t)+u^{\mathrm{T}}(t) u(t) \\
= & -u^{\mathrm{T}}(t) u(t)+u^{\mathrm{T}}(t) u(t)+u^{\mathrm{T}}(t) u(t) \\
= & u^{\mathrm{T}}(t) u(t) .
\end{aligned}
$$

Because $u(t)$ is a continuous function, $r(u(t), y(t))$ is Lebesgue integrable. However, if we choose

$$
Q=\left[\begin{array}{cc}
-1 & 0 \\
0 & 0
\end{array}\right] \leq 0, \quad S=\left[\begin{array}{l}
0 \\
1
\end{array}\right], \quad R=1,
$$

they will not satisfy conditions in Theorem 12 . In this case, we can verify that

$$
\begin{aligned}
r(u(t), y(t))= & y^{\mathrm{T}}(t) Q y(t)+2 u(t)^{\mathrm{T}} S y(t)+u^{\mathrm{T}}(t) R u(t) \\
= & -x^{\mathrm{T}}(t) C^{\mathrm{T}} Q C x(t)+2 x^{\mathrm{T}}(t) C^{\mathrm{T}} S u(t) \\
& +u^{\mathrm{T}}(t) R u(t) \\
= & -x_{1}^{\mathrm{T}}(t) x_{1}(t)+2 x_{3}^{\mathrm{T}}(t) u(t)+u^{\mathrm{T}}(t) u(t) \\
= & -x_{1}^{\mathrm{T}}(t) x_{1}(t)+3 u^{\mathrm{T}}(t) u(t)
\end{aligned}
$$

is not Lebesgue integrable as it will contain $\delta(t-\beta) \delta(t-\beta)$ which is not integrable.

Example 2 verifies the validity of Theorem 12 . When a descriptor system has impulsive behavior, the supply function unlike impulse-free system is not always Lebesgue integrable. In this case, we need to choose the parameters $(Q, S, R)$ carefully in order to guarantee dissipativity.

\section{Conclusions}

In this paper, we investigate the dissipativity problem for continuous descriptor systems. A new image space is used to characterize the state responses of descriptor systems with impulsive behavior. Following it, we give a dissipativity theorem for descriptor systems in general. This result is valid regardless of the impulsive behavior, which is different from the existing results. The integrability of a supply function and the parametrization form of all solutions for the proposed LMIs are discussed. Meanwhile, our results include some previous ones as special cases. Finally, some examples are given to show validity of the results obtained in this paper.

The results in this paper are mainly based on analysis. It should be pointed out that the image space approach can also be used to solve control/synthesis problems for descriptor systems. Some related problems are under our current investigation.

Another significant issue in this paper is the choice of delta function using the limit of a series of normal functions. It is our task in the future to fill this gap by using the generalized function theory though we believe all the results derived in Lemma 8 and Theorem 12 are true in general.

\section{Conflict of Interests}

The authors declare that there is no conflict of interests regarding the publication of this paper.

\section{Acknowledgments}

The authors thank all the reviewers and the Associate Editor for their constructive comments as the quality of this paper is improved significantly after significant revision based on their comments. This work was supported by National Natural Science Foundation of China (61273008, 60974004, and 61104003).

\section{References}

[1] J. C. Willems, "Dissipative dynamical systems: part I. General theory," Archive for Rational Mechanics and Analysis, vol. 45, no. 5, pp. 321-351, 1972.

[2] D. J. Hill and P. J. Moylan, "Connections between finite-gain and asymptotic stability," IEEE Transactions on Automatic Control, vol. 25 , no. 5, pp. 931-936, 1980.

[3] D. J. Hill and P. J. Moylan, "Stability results for nonlinear feedback systems," Automatica, vol. 13, no. 4, pp. 377-382, 1977.

[4] B. Brogliato, R. Lozano, and O. Egeland, Dissipative Systems Analysis and Control: Theory and application, Communications 
and Control Engineering Series, Springer, London, UK, 2nd edition, 2000.

[5] J. C. Willems, "Dissipative dynamical systems part II: linear systems with quadratic supply rates," Archive for Rational Mechanics and Analysis, vol. 45, no. 5, pp. 352-393, 1972.

[6] S. Xie, L. Xie, and C. E. de Souza, "Robust dissipative control for linear systems with dissipative uncertainty," International Journal of Control, vol. 70, no. 2, pp. 169-191, 1998.

[7] Z. Q. Tan, Y. C. Soh, and L. H. Xie, "Dissipative control for linear discrete-time systems," Automatica, vol. 35, no. 9, pp. 1557-1564, 1999.

[8] Z. Li, J. Wang, and H. Shao, "Delay-dependent dissipative control for linear time-delay systems," Journal of the Franklin Institute, vol. 339, no. 6-7, pp. 529-542, 2002.

[9] M. Meisami-Azad, J. Mohammadpour, and K. M. Grigoriadis, "Dissipative analysis and control of state-space symmetric systems," Automatica, vol. 45, no. 6, pp. 1574-1579, 2009.

[10] S. Gupta, "Robust stabilization of uncertain systems based on energy dissipation concepts," National Aeronautics and Space Administration Contractor Report 4713, 1996.

[11] L. Dai, Singular Control Systems, vol. 118 of Lecture Notes in Control and Information Sciences, Springer, 1989.

[12] C. Y. Yang, Q. L. Zhang, Y. P. Lin, and L. Zhou, "Positive realness and absolute stability problem of descriptor systems," IEEE Transactions on Circuits and Systems I: Regular Papers, vol. 54, no. 5, pp. 1142-1149, 2007.

[13] D. Cobb, "Controllability, observability, and duality in singular systems," IEEE Transactions on Automatic Control, vol. 29, no. 12, pp. 1076-1082, 1984.

[14] M. S. Mahmoud and H. E. Emara-Shabaik, "New $H_{2}$ filter for uncertain singular systems using strict LMIs," Circuits, Systems, and Signal Processing, vol. 28, no. 5, pp. 665-677, 2009.

[15] S. Y. Xu, J. Lam, and C. Yang, "Robust $H_{\infty}$ control for discrete singular systems with state delay and parameter uncertainty," Dynamics of Continuous, Discrete and Impulsive Systems B: Applications \& Algorithms, vol. 9, no. 4, pp. 539-554, 2002.

[16] J. Ren and Q. Zhang, "Robust $H_{\infty}$ control for uncertain descriptor systems by proportional-derivative state feedback," International Journal of Control, vol. 83, no. 1, pp. 89-96, 2010.

[17] E. Zeheb, R. Shorten, and S. S. K. Sajja, "Strict positive realness of descriptor systems in state space," International Journal of Control, vol. 83, no. 9, pp. 1799-1809, 2010.

[18] M. K. Camlibel and R. Frasca, "Extension of KalmanYakubovich-Popov lemma to descriptor systems," Systems \& Control Letters, vol. 58, no. 12, pp. 795-803, 2009.

[19] Y. Gao, G. Lu, and Z. Wang, "Passive control for continuous singular systems with non-linear perturbations," IET Control Theory \& Applications, vol. 4, no. 11, pp. 2554-2564, 2010.

[20] X. Z. Dong and Q. L. Zhang, "Robust strictly dissipative control for linear singular systems," Control and Decision, vol. 20, no. 2, pp. 195-198, 2005.

[21] I. Masubuchi, "Dissipativity inequalities for continuous-time descriptor systems with applications to synthesis of control gains," Systems \& Control Letters, vol. 55, no. 2, pp. 158-164, 2006.

[22] I. Masubuchi, "Output feedback controller synthesis for descriptor systems satisfying closed-loop dissipativity," Automatica, vol. 43, no. 2, pp. 339-345, 2007.

[23] X. Z. Dong, "Robust strictly dissipative control for discrete singular systems," IET Control Theory \& Applications, vol. 1, no. 4, pp. 1060-1067, 2007.
[24] C. Han, L. Wu, P. Shi, and Q. Zeng, "On dissipativity of TakagiSugeno fuzzy descriptor systems with time-delay," Journal of the Franklin Institute, vol. 349, no. 10, pp. 3170-3184, 2012.

[25] H. Gassara, A. El Hajjaji, M. Kchaou, and M. Chaabane, "Observer based $(Q, V, R)$ - $\alpha$-dissipative control for TS fuzzy descriptor systems with time delay," Journal of the Franklin Institute, vol. 351, no. 1, pp. 187-206, 2014.

[26] W. X. Cui, J. A. Fang, Y. L. Shen, and W. B. Zhang, "Dissipativity analysis of singular systems with Markovian jump parameters and mode-dependent mixed time-delays," Neurocomputing, vol. 110, pp. 121-127, 2013.

[27] D. Chu and R. C. E. Tan, "Algebraic characterizations for positive realness of descriptor systems," SIAM Journal on Matrix Analysis and Applications, vol. 30, no. 1, pp. 197-222, 2008.

[28] W. Q. Liu, W. Y. Yan, and K. L. Teo, "On initial instantaneous jumps of singular systems," IEEE Transactions on Automatic Control, vol. 40, no. 9, pp. 1650-1655, 1995.

[29] Q. L. Zhang and D. M. Yang, Analysis and Synthesis for Uncertain Descrptor Systems, Northeastern University Press, Shenyang, China, 2003.

[30] G.-R. Duan, Analysis and Design of Descriptor Linear Systems, vol. 23 of Advances in Mechanics and Mathematics, Springer, New York, NY, USA, 2010.

[31] K. D. Hjelmstad, Fundamentals of Structural Mechanics, Springer, 2nd edition, 2004.

[32] G. B. Arfken and H. J. Weber, Mathematical Methods for Physicists, Academic Press, Boston, Mass, USA, 5th edition, 2000.

[33] J. Löfberg, "YALMIP: a toolbox for modeling and optimization in MATLAB," in Proceedings of the IEEE International Symposium on Computer Aided Control System Design, pp. 284-289, Taipei, Taiwan, September 2004.

[34] J. F. Sturm, "Using SeDuMi 1.02, a Matlab toolbox for optimization over symmetric cones," Optimization Methods and Software, vol. 11, no. 1-4, pp. 625-653, 1999. 


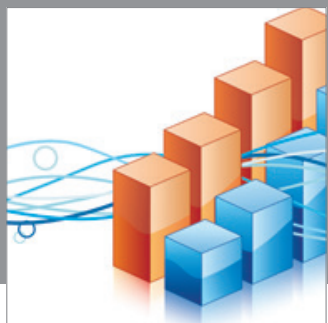

Advances in

Operations Research

mansans

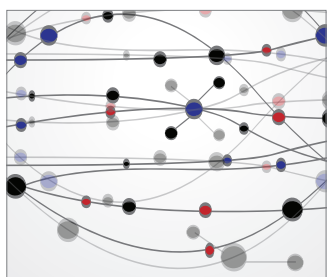

The Scientific World Journal
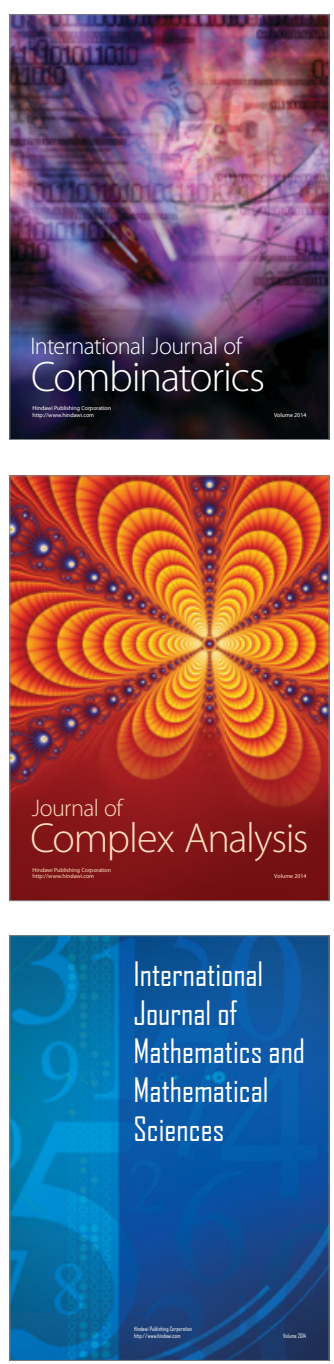
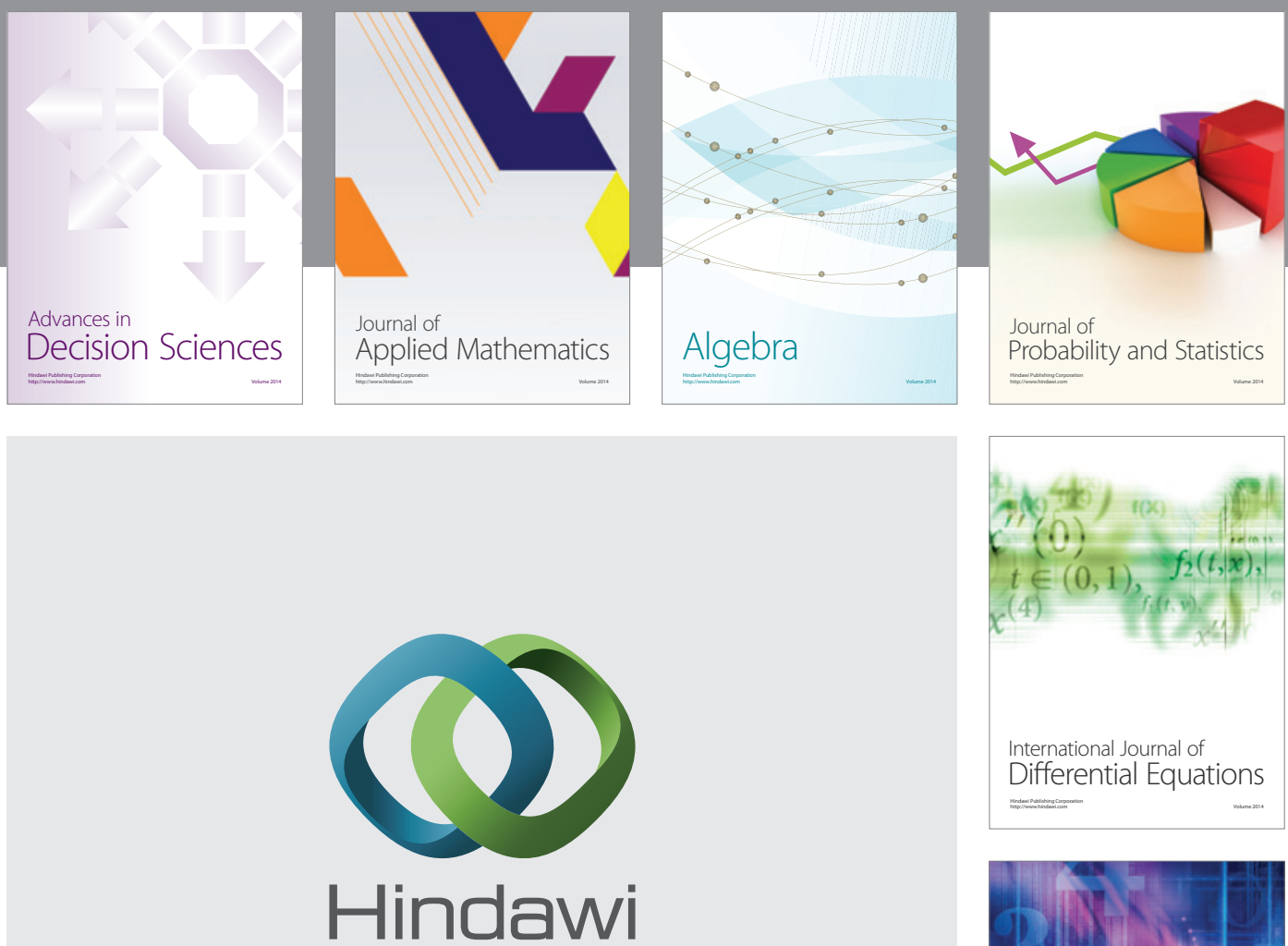

Submit your manuscripts at http://www.hindawi.com
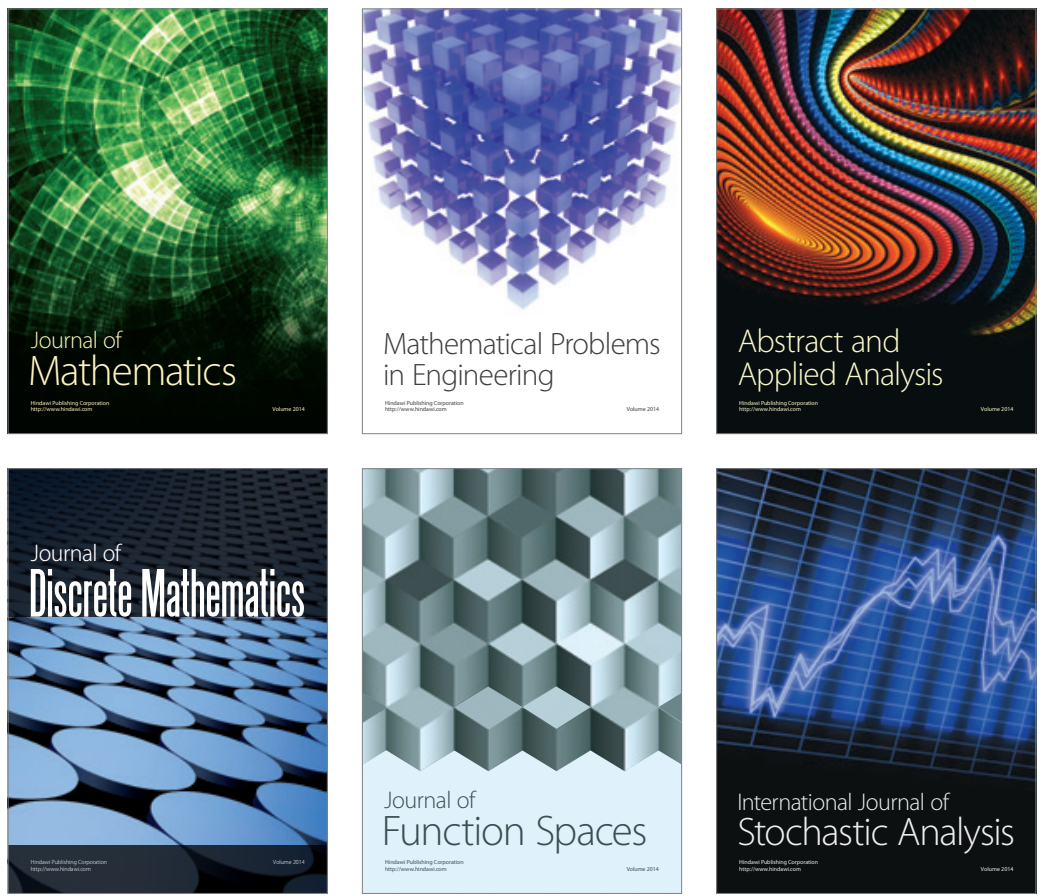

Journal of

Function Spaces

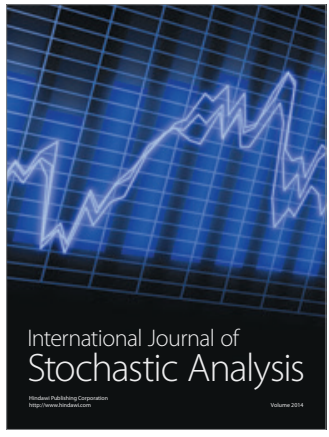

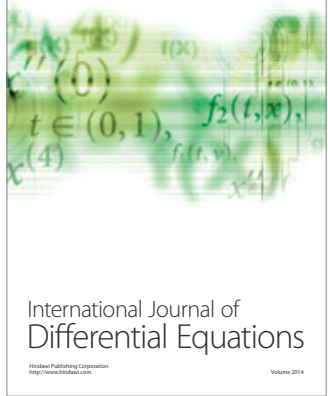
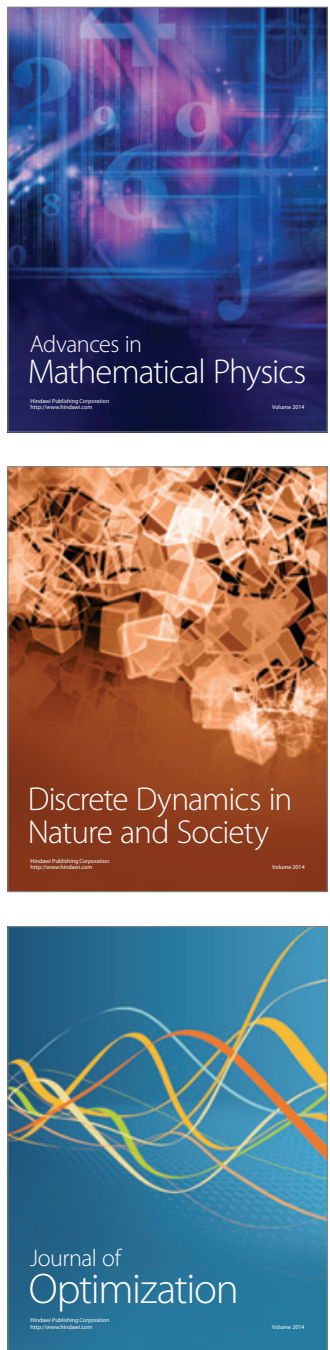